\section{Summit of the Research Coordination Networks for Undergraduate Biology Education}

\author{
Carrie Diaz Eaton, ${ }^{\dagger}$ Deborah Allen, ${ }^{\ddagger}$ Laurel J. Anderson, ${ }^{\S}$ Gillian Bowser," \\ Mark A. Pauley, " Kathy S. Williams," and Gordon E. Uno ${ }^{\text {e* }}$ \\ ${ }^{\dagger}$ Center for Biodiversity, Unity College, Unity, ME 04988; 'Department of Biological Sciences, \\ University of Delaware, Newark, DE 19716; 'Department of Botany \& Microbiology, Ohio \\ Wesleyan University, Delaware, $\mathrm{OH} 43015$; "Department of Ecosystem Science and Sustainability, \\ Colorado State University, Fort Collins, CO 80523; 'School of Interdisciplinary Informatics, \\ University of Nebraska at Omaha, Omaha, NE 68182; "Department of Biology, San Diego State \\ University, San Diego, CA 92182; ‘ Department of Microbiology and Plant Biology, University of \\ Oklahoma, Norman, OK 73019
}

\begin{abstract}
The first summit of projects funded by the National Science Foundation's Research Coordination Networks for Undergraduate Biology Education (RCN-UBE) program was held January 14-16, 2016, in Washington, DC. Sixty-five scientists and science educators from 38 of the 41 Incubator and Full RCN-UBE awards discussed the value and contributions of RCNs to the national biology education reform effort. The summit illustrated the progress of this innovative UBE track, first awarded in 2009. Participants shared experiences regarding network development and growth, identified best practices and challenges faced in network management, and discussed work accomplished. We report here on key aspects of network evaluation, characteristics of successful networks, and how to sustain and broaden participation in networks. Evidence from successful networks indicates that 5 years (the length of a Full RCN-UBE) may be insufficient time to produce a cohesive and effective network. While online communication promotes the activities of a network and disseminates effective practices, face-to-face meetings are critical for establishing ties between network participants. Creation of these National Science Foundation-funded networks may be particularly useful for consortia of faculty working to address problems or exchange novel solutions discovered while introducing active-learning methods and/ or course-based research into their curricula.
\end{abstract}

\section{INTRODUCTION}

There is a growing body of diverse examples that demonstrate how well-designed networks enhance the flow of ideas and lead to innovation (Pentland, 2014). Networking to communicate research strategies and outcomes is now an essential aspect of the process of science (Schmidt, 2015). Supporting that notion, the National Science Foundation (NSF) initiated the Research Coordination Network (RCN) program in 2000. The RCN program funds projects in basic research or education that aim to promote a field or inspire new directions through increased collaborative work by network members. The intended outcome of an RCN is not to produce research products per se, but rather to create opportunities to nurture new collaborations and address novel research topics that arise through interactions with colleagues. Advances in biology education are being made using a wide variety of strategies that span disciplinary, organizational, and geographic boundaries. Thus, in 2009, NSF made the first RCN awards for biology education projects, using a dedicated track within the program, the RCN-UBE (Undergraduate Biology Education) track, to "catalyze positive changes in biology undergraduate education" (NSF, 2008).
CBE Life Sci Educ December 1, 2016 15:mr1 DOI:10.1187/cbe.16-03-0147

*Address correspondence to: Gordon E. Uno (guno@ou.edu).

(C) 2016 C. D. Eaton et al. CBE-Life Sciences Education (C) 2016 The American Society for Cell Biology. This article is distributed by The American Society for Cell Biology under license from the author(s). It is available to the public under an Attribution-Noncommercial-Share Alike 3.0 Unported Creative Commons License (http://creativecommons.org/licenses/ by-nc-sa/3.0)

"ASCB®" and "The American Society for Cell Biology ${ }^{\circledR}$ " are registered trademarks of The American Society for Cell Biology. 
RCN funds can be used to support new collaborations and are designed to spur networks that will share information and, as a result, advance the field. (RCN awards do not support existing networks or ongoing collaborations.) Funded networking activities may include support for travel and/or meetings to share information, coordinating planned research, synthesizing knowledge, and/or developing community standards and assessments. Funds can also be used to pay for support staff or the time of a coordinator to organize and lead activities of the network and for virtual infrastructure to support ongoing collaboration, outreach, and dissemination. However, funds may not be used to support the research collaborations that result. The RCN-UBE track is unique in the RCN program, in that it offers both 1-year "Incubator" awards to seed network development and 5-year "Full" awards. As of NSF fiscal year 2016, 41 RCN-UBE projects have been funded, either as Incubators or as Full awards; several Incubator awardees reapplied to the program and were awarded a Full project (Table 1).

The 41 NSF RCN-UBE-funded networks are using a diverse group of strategies, all aimed at improving undergraduate biology education, but all unique (Table 1). Researchers from RCNs in other multidisciplinary research fields (such as ecology) have sometimes formed broader collaborative communities through regular meetings. For example, members of the Long Term Ecological Reserves (LTER) program and of the Field Stations and Marine Laboratories (FSML) program meet regularly, sometimes formally (e.g., through the Organization of Biological Field Stations, OBFS). These gatherings of networked "communities" have provided opportunities for scientists, often with diverse backgrounds, to connect, communicate, and develop collaborations that can lead to new research trajectories. Owing to our common theme of undergraduate biology education, many RCNUBE networks share members but also include those spanning different areas of expertise. Thus, it seemed very worthwhile to assemble an "RCN community," where the individual RCN-UBE networks could share information on such common issues as promoting diversity, supporting multidisciplinary collaboration, and identifying ways to sustain their efforts, among others. Such a meeting of the leadership of the RCN-UBE networks could open up new possibilities for innovation in biology education and would allow us to target opportunities in ways a single RCN-UBE might not be able to do (National Research Council, 2014).

Although RCN principal investigators (PIs) have convened on several occasions to discuss common challenges and opportunities in building, sustaining, and managing RCNs, the last of these meetings was in December 2010, a time when the RCNUBE program was too new for meaningful exchanges specific to the track. Given that there is now a vibrant community, a group of RCN-UBE PIs organized an RCN-UBE Summit, which was held January 14-16, 2016, in Washington, DC, with support from the NSF. Sixty-five people, representing 38 RCN-UBE projects (Table 1), attended the meeting.

In addition to highlighting and reviewing the progress of the RCN-UBE projects, the goals of the meeting included identifying ways to improve, grow, sustain, and evaluate the individual networks and the RCN-UBE program itself. The summit also enabled participants to build connections that could potentially develop into novel collaborations across the RCN-UBE community. Presentations, panel discussion sessions, and two poster sessions, provided attendees with opportunities to learn about the activities of the RCNs represented at the summit. Finally, we explored ways to disseminate strategies for network growth, maintenance, and evaluation that have been tried and adopted by the many types of networks represented at the summit.

Summit activities were organized around the following themes: 1) sharing successes achieved by diverse RCN-UBEs, 2) exploring successful strategies for network management, 3) broadening participation in networks, 4) sustaining networks through time, and 5) evaluating network success. Each was discussed during different meeting sessions. Summaries of findings and recommendations from each session are presented below.

\section{Sharing Successes}

A full list of the networks represented at the RCN-UBE Summit appears in Table 1. Examples of RCNs formed with support of the RCN-UBE program include: 1) Course-based Undergraduate Research Experiences Network (CUREnet; http://curenet .cns.utexas.edu), which is creating course-based undergraduate research experiences in biology with the goal of fostering students' understanding of core biology concepts and development of scientific competencies; 2) an online networking hub for collaboration, discovery, and synthesis in quantitative biology curricula, now called QUBES, for Quantitative Undergraduate Biology Education and Synthesis (2015; http://qubeshub.org), which is bringing together mathematics and biology; 3) Network for Integrating Bioinformatics into Life Sciences Education (NIBLSE, 2016; "nibbles"; http://qubeshub.org/groups/niblse), which is working to integrate the emerging field of bioinformatics into the life sciences curriculum; 4) Ecological Research as Education Network (EREN, 2010; http://erenweb.org), which is developing educational activities anchored in authentic, collaborative, ecological research experiences; 5) Faculty Developer Network for Undergraduate Biology Education (FDN-UBE; http://biofacdevelopers.org), which is addressing challenges in motivating, sustaining, and supporting faculty change toward reform-based teaching practices; and 6) the Rocky Mountain Sustainability and Science Network (RMSSN), which focuses on undergraduate learning in informal settings and has transitioned from public to sustained financing through a nonprofit model. In addition to the networks listed in Table 1, many other successful biology education networks exist-for example, HHMI SEA-PHAGES (www.hhmi.org/programs/science-education-alliance), the Genomics Education Partnership (GEP; https://gep.wustl.edu), BioQUEST Curriculum Consortium (http://bioquest.org), and the Genome Consortium for Active Teaching (GCAT; http://bio.davidson.edu/gcat). Because these networks were started without NSF RCN support, they were not represented at this summit.

\section{Characteristics of Successful Networks: Network Management}

The summit opened with a discussion of key characteristics of successful networks. This was informed by an informal survey of 87 participants at two meetings of the RCN-UBE "Preparing to Prepare the 21st Century Biology Student" (Table 1). Participants were asked for their responses to the question "What makes a network work?" The following key features of successful networks were identified:

1. Shared goals and visions are critical. Successful networks consist of people with similar aims who are facing similar 
TABLE 1. Projects represented by the attendees of the first summit meeting of the RCN-UBE, a track within NSF's RCN program

\begin{tabular}{|c|c|c|}
\hline Award year & Principal investigator & Project title (award type: Incubator [I] or Full $[\mathrm{F}])^{\mathrm{a}}$ \\
\hline 2009 & Gordon E. Uno & $\begin{array}{l}\text { Preparing to Prepare the 21st Century Biology Student: Using Scientific Societies as } \\
\text { Change Agents for the Introductory Biology Experience }[\mathrm{F}]\end{array}$ \\
\hline 2009 & Patricia Harrison & $\begin{array}{l}\text { Open Science: An Education Network in Ethnobiology to Coordinate the } \\
\text { Development of a New Culture in the Undergraduate Science Classroom [F] }\end{array}$ \\
\hline 2009 & Christopher Kvaal & $\begin{array}{l}\text { RCN-UBE Collaborative Workshop held Friday, January 30, to Saturday, January } \\
\text { 31, 2009, in Minneapolis, MN [I] }\end{array}$ \\
\hline 2010 & Lori Scott & Microbial Genome Annotation Network $[\mathrm{F}]$ \\
\hline 2010 & Laurel J. Anderson & $\begin{array}{l}\text { Establishing an Ecological Research/Education Network at Primarily Undergraduate } \\
\text { Institutions }[\mathrm{F}]\end{array}$ \\
\hline 2010, 2012 & Mary Pat Wenderoth & $\begin{array}{l}\text { SABER: Accelerating the Emergence of Biology Education Research as a New } \\
\text { Subdiscipline of Biology [I, F] }\end{array}$ \\
\hline 2010 & Gillian Bowser & $\begin{array}{l}\text { Rocky Mountain Sustainability and Science Network: Enhancing Undergraduate } \\
\text { Student Learning of Biological Concepts }[\mathrm{F}]\end{array}$ \\
\hline 2010 & Joseph Cook & Advancing Integration of Museums into Undergraduate Programs (AIM-UP!) [F] \\
\hline 2010 & Wendy Silk & Trial Network to Bring Music to the Study of Biology [I] \\
\hline 2010 & Lee Hughes & $\begin{array}{l}\text { Planning a Research Network on Assessing Learning Technologies in Undergraduate } \\
\text { Biology Education [I] }\end{array}$ \\
\hline 2010 & Ellis Bell & $\begin{array}{l}\text { Promoting Concept-Driven Teaching Strategies in Biochemistry and Molecular } \\
\text { Biology through Concept Assessments [F] }\end{array}$ \\
\hline 2010 & Marlene Moore & $\begin{array}{l}\text { Willamette Valley Biological Education Network (WVBEN): Using Regional } \\
\text { Pedagogical Communities to Improve Undergraduate Learning Environments in } \\
\text { Biology [I] }\end{array}$ \\
\hline 2010 & Joseph Koonce & Lake Erie Watershed Research and Education Network [I] \\
\hline 2010, 2011 & Margaret Waterman & The Case Study and PBL Network [I, F] \\
\hline 2010, 2011 & $\begin{array}{l}\text { Kathleen Fisher and Kathy } \\
\text { S. Williams }\end{array}$ & $\begin{array}{l}\text { BioHUB: An Internet HUB for the Conceptual Assessment in Biology Community } \\
\text { [I, F] }\end{array}$ \\
\hline 2011, 2014 & Rachelle Spell & $\begin{array}{l}\text { Bridging the Divide between Research and Education with Authentic Research } \\
\text { Experiences in Introductory Biology [I, F] }\end{array}$ \\
\hline 2011 & Erin Dolan & Course-based Undergraduate Research Experiences Network (CUREnet) [F] \\
\hline 2011 & Michael Boyle & $\begin{array}{l}\text { Transforming Undergraduate Education through Increased Faculty Access to } \\
\text { NextGen Sequencing Runs [I] }\end{array}$ \\
\hline 2011 & Holly Gaff & Interdisciplinary Communication Laboratory for Undergraduate Biology (iCLUB) [I] \\
\hline 2011 & Raphael Isokpehi & Visual Analytics in a Biology Curriculum Network [I] \\
\hline 2013 & Philip Myers & Enhancing Data Discovery and Usability for Inquiry in Biology Education [I] \\
\hline 2013, 2015 & Elisabeth Schussler & Biology Teaching Assistant Project (BioTAP) [I, F] \\
\hline 2013 & Vincent Buonaccorsi & $\begin{array}{l}\text { GCAT-SEEK: The Genome Consortium for Active Undergraduate Research and } \\
\text { Teaching Using Next-Generation Sequencing }[\mathrm{F}]\end{array}$ \\
\hline 2013 & Susan Keen & $\begin{array}{l}\text { Animated Discussions: Biologists and Visual Artists Foster Learning through } \\
\text { Animations [I] }\end{array}$ \\
\hline 2013 & Stasinos Stavrianeas & $\begin{array}{l}\text { Northwest Biosciences Consortium (NWBC): Implementation of Vision and Change } \\
\text { in the Introductory Biology Curriculum }[\mathrm{F}]\end{array}$ \\
\hline 2014 & Marsha Matyas & Growing a Physiology Education Community of Practice [I] \\
\hline 2014 & Diann Jordan & $\begin{array}{l}\text { HBCU-ALBN: Network of Biologists and Life Science Educators for Vision and } \\
\text { Change in the Curriculum [I] }\end{array}$ \\
\hline 2014, 2015 & Mark A. Pauley & Network for Integrating Bioinformatics into Life Sciences Education (NIBLSE) [I, F] \\
\hline 2014 & Nancy Palaez & $\begin{array}{l}\text { Assessment of Competence in Experimental Design in Biology (ACED-Bio) Network } \\
{[\mathrm{F}]}\end{array}$ \\
\hline 2014 & Robert Newman & $\begin{array}{l}\text { Development of a Build-a-Genome Network to Teach Synthetic Biology at Diverse } \\
\text { Undergraduate Institutions [I] }\end{array}$ \\
\hline 2014 & Michael LaMar & $\begin{array}{l}\text { An Online Networking Hub for Collaboration, Discovery, and Synthesis in } \\
\text { Quantitative Biology Curricula [I] }\end{array}$ \\
\hline 2014 & Deborah Allen & Faculty Development Network for Undergraduate Biology (FDN-UB) [F] \\
\hline 2014 & William Davis & $\begin{array}{l}\text { Northwest PULSE: A Community of Practice for Departmental Transformation } \\
\text { Using Vision and Change }[\mathrm{F}]\end{array}$ \\
\hline 2015 & Sue Wick & Promoting Active Learning and Mentoring (PALM) [I] \\
\hline 2015 & Teresa Mourad & Next Generation Careers-Innovation in Environmental Biology Education [I] \\
\hline 2015 & Gordon E. Uno & $\begin{array}{l}\text { RCN-UBE Summit: Learning from the Community of Education Networks } \\
\text { [conference] }\end{array}$ \\
\hline
\end{tabular}

an cases in which an Incubator project [I] was followed by a Full award [F], the title is that of the Full award. 
challenges, but may also include people from different disciplines.

2. Networks should have effective and regular communication. It is helpful for network members to receive regular updates about network activities; for example, through webinars and meetings or scheduled conference calls and email.

3. Networks should be a safe place to share and discuss ideas. The environment should be collaborative, constructive, and positive, so that there is trust among members.

4. Although online meetings can be used to reinforce connections, face-to-face time is essential for effective collaboration.

5. There should be easy access to forums and an engaging project website. These should provide opportunities for members to contribute asynchronously. Some networks use social media effectively in this context.

6. It is important to cultivate interpersonal relationships in the network so that participants want to interact and support one another.

7. There should be mechanisms to efficiently share resources, such as a repository of strategies and methods, accompanied by a framework to encourage frequent contributions and discussion.

8. Successful networks have people who can bring ideas into practice. Successful UBE networks have members who have specific expertise (of various types) relevant to improving science education who can share that knowledge.

9. Networks work best when there are many people involved with a high proportion of members actively participating. Consequently, mechanisms have to be developed for dealing with people who do not contribute.

10. Basic group norms should be established; clear and mutually accepted formats for interactions, relevant activities, and functions will facilitate collaborations.

11. Every RCN benefits from a point person to keep the group going; that is, a nexus/taskmaster to focus and organize efforts, who maintains a timeline and sets deadlines.

Participants agreed that these characteristics of successful networks, with specific examples from RCN-UBEs of how these best practices were carried out, formed the basis for useful network management. Slides for a related discussion of network management are available at the summit website (RCN-UBE Summit, 2016).

\section{RCN-UBE CHALLENGES AND OPPORTUNITIES Broadening Participation in Networks}

There was a strong common desire at the summit to increase the number of faculty and students from underrepresented groups who are participating in and benefiting from RCN-UBE activities. Panelists in this session included discussants from Howard University, a historically Black college in Washington, DC, and leaders from RCNs such as the RMSSN (Table 1), which have had success in recruiting diverse student and faculty participants. To effectively broaden participation in RCNs, more faculty and researchers from diverse backgrounds and with a variety of experiences need to be recruited as active members with meaningful roles. Dr. George Middendorf from
Howard University highlighted the importance of bringing historically Black colleges and universities (HBCU) members in as full partners at the beginning of project development, not after the RCN itself has been formed. Dr. Gillian Bowser, representing RMSSN (Table 1), discussed perceived recruitment barriers for underrepresented minority (URM) students and the importance of approaching recruitment from the perspective of URM students themselves. Presenting the successful recruitment practices of RMSSN as an example, Dr. Bowser highlighted using student voices as part of the recruitment strategy for RMSSN, for which applicant and recruitment pools regularly exceed $80 \%$ URM students despite the lead institution, Colorado State University, being predominantly white. Listening to the voices of the URM students by using self-efficacy survey techniques from the social sciences is an innovative approach to broadening participation in the ecological sciences; during the past two decades, self-efficacy has emerged as a highly effective predictor of students' motivation and learning (Zimmerman, 2000). RCN-UBE leaders agreed that reaching out to faculty at 2-year and minority-serving institutions might help with diversity goals but that more effort was needed to change recruitment styles to a focus on the perceptions and motivation of the minority students themselves. In sum, successful strategies for broadening minority participation in networks include bringing diverse faculty and institutions into the RCNs in a meaningful way, starting at the network's inception. Equally important is the use of techniques from other disciplines, such as the social sciences, to understand perceptions of barriers that URM students, faculty, and researchers face in the sciences and to consider how RCN approaches can overcome such perceptions and provide a welcoming space.

\section{Sustaining Networks through Time}

Evidence from the RCN-UBE projects and remarks by the speakers at the summit suggested that a 5-year award may not provide sufficient time for most RCN-UBE networks to reach their full potential. Information shared at the summit indicated that many RCNs required more than 5 years for members to form collaborations robust enough to affect scientific output. Thus, it was proposed that the RCN-UBE program adopt a mechanism by which "mature" RCN-UBE projects-those that have demonstrated success and progress toward their goals-could seek and receive additional funding to complete project implementation, find ways to sustain themselves, or develop new projects that allow them to evolve in sustainable directions.

As discussed at the summit, some RCN-UBEs have creatively moved into other realms as a way to evolve beyond RCN funding. For example, some have pursued support from other funding programs (e.g., QUBES was awarded an NSF Improving Undergraduate STEM Education: Education and Human Resources [IUSE] Ideas Lab grant), become professional societies (e.g., the establishment of the Society for the Advancement of Biology Education Research [SABER]), or formed nonprofit organizations (e.g., the RMSSN, which has received private foundation funding). RCN-UBE PIs have begun discussing ways to identify and share information about these opportunities for sustaining their communities beyond RCN-UBE funding. In addition, new projects and collaborations have emerged from the discussions and interactions that occurred at RCN-UBE 
meetings. For instance, the new FDN-UBE emerged after discussions held at meetings of the "Preparing to Prepare the 21st Century Biology Student” UBE (Table 1).

\section{Evaluating Network Success}

NSF programs such as the earlier Transforming Undergraduate Education in STEM (TUES) and the current IUSE programs have produced traditional publication products describing their innovations and findings. In contrast, RCNs are measured by how successfully they build networks of research collaborators and participants that expand research horizons, a very different outcome from other funded projects. This is an advantage, because the RCN-UBEs can focus on developing strong coalitions in biology education (coupled with other disciplines, such as the social sciences, as appropriate) that can drive widespread and sustainable change. One example of such an RCN-UBE project evolving into a meaningful voice in biology education is SABER, now a national society that had more than 500 registrants for the Fifth Annual Meeting in 2015 (SABER, 2016). Another example is the EREN project, an RCN that facilitates faculty and undergraduate student participation in multisite ecological research (Bowne et al., 2011; Simmons et al., 2016). This project has engaged more than 300 faculty members from more than 200 institutions (with some international partners) and has reached an estimated $4000+$ undergraduate students. EREN enables participants from primarily undergraduate institutions to engage in ecological studies at larger spatial scales than most faculty and students at these institutions can address alone. However, largely due to the nature of long-term multisite ecological research and the complexity of managing a multisite project with multiple collaborators (many of whom have primarily teaching roles with limited time for research), the production of science publications is often slow. Engaging students in authentic undergraduate research opportunities is considered a high-impact practice for student success (Kuh, 2008). However, obtaining funding to manage coordination of such opportunities is difficult when publications are produced on a slower timeline and on a smaller scale than would be expected of a conventional research laboratory. EREN has helped its member faculty engage in these activities without adding the pressure of needing to publish a high number of research papers. (See the summit website [RCN-UBE Summit, 2016] for the presentation about EREN.) Finally, RMSSN is an example of a network that has successfully tracked participating students over the course of the project. RMSSN maintains communication with members of every class since 2009. RMSSN is a smaller RCN, with faculty participants from 14 institutions (including community colleges, minority-serving institutions, and international institutions) mentoring just over 200 student participants.

Formal project evaluation generally uses multiple alternative approaches to measure impact, and the summit benefited from program assessment advice presented by Pamela Bishop (associate director for science, technology, engineering, and mathematics (STEM) evaluation at the National Institute for Mathematical Biology and Synthesis [NIMBioS]), Yolanda George (American Association for the Advancement of Science), and Chris Meyer (NSF). Social networking analysis and other approaches from the social sciences are being applied to this area in novel ways and may be adopted for describing RCNs. Unfortunately, RCN budgets, particularly those for Incubator-level projects, are often not sufficient to provide incentives for participants or to support crucial elements of a project, such as an external evaluator. Leveraging campus resources and online prototypes for assessment can be helpful. Small groups discussed the use of survey instruments to collect data on various aspects of our diverse projects to measure their activities and outcomes. A working group has begun to construct and pilot a survey using measures identified at the summit that would describe these varied UBE networks and projects.

Clearly, all summit participants recognized the value of program assessment for RCN-UBEs and encouraged the application of strong assessment plans for all long-term education projects. A white paper or miniconference about evaluating network education projects could be a valuable future activity of the RCN-UBE program.

\section{A NETWORK OF NETWORKS}

The summit was an excellent opportunity for RCNs to learn from one another, especially for the newest projects to learn from the experiences of more mature RCNs. The RCN-UBE community is developing strategies to continue communications and networking. Participants felt that their interactions with colleagues were highly valuable; all participants met new colleagues, and many developed nascent collaborations. These face-to-face interactions are critical to the formation of any network and to future collaborative work. Future meetings of RCN-UBE PIs will piggyback on the next Gordon Research Conference on Undergraduate Biology Education Research (Summer 2017) or similar national meetings. This will allow summit participants to engage others interested in becoming part of their network or developing new networks, whether or not they are funded by the RCN-UBE program.

To preserve interactions and promote future ones, QUBES developed a website for the summit that provides the infrastructure for collaboration (https://qubeshub.org/groups/rcn ube2016). All interested parties are welcome to participate. Unlike a digital repository, QUBES infrastructure is designed for collaboration and informal sharing of ideas and resources and for partners with existing digital repositories to further disseminate good work into biology education networks (Donovan et al., 2015). We encourage the community at large to become an active part of this conversation around biology education. QUBES invited all RCN-UBE projects to create and use space and offered assistance to do so. (While getting into QUBES requires a log-in, access to the site and use of space is free of charge to non-RCN science education projects, although contributions to the hub are welcomed.) Some current projects, such as EREN and BioHUB (a resource for conceptual assessments in biology), are creating groups on QUBES hub to utilize the database and data analytics features, while others are using group space for organization of meetings and materials (e.g., NIBLSE, 2016).

\section{RECOMMENDATIONS}

Recommended practices and strategies for achieving our goals were discussed extensively at the summit, resulting in the 
"Network Management" list presented above. Systematically sharing information about which strategies work and which do not is an essential benefit of networking across all RCNUBE communities that needs to be sustained. We note that evaluating success of these projects requires evaluation of contributions other than traditional products; this will require the identification and use of diverse measures. Clearly, community building and productively addressing the diversity and inclusivity of these communities is a much-desired outcome and will take time to accomplish. Therefore, summit participants are encouraging funding agencies to provide support both for appropriate program-level assessment and evaluation and for funding durations to support the time required to nurture the growth of communities and identify alternative resources to sustain that growth to achieve a sustainable mode of operation.

In summary, the RCN-UBE community, as members of the growing biology education community, aims to help distribute knowledge about excellent biology and STEM education activities and resources to stakeholders in the public, private, and policy-making sectors. For those readers considering submitting an RCN-UBE grant proposal to spur new collaborations to synthesize and advance knowledge in biology education, we offer a few suggestions: 1) bring all your partners into the planning process early as true collaborators with a shared and unifying vision; 2) include diverse partners and institutions at the start of the project; 3) make room in the budget for a coordinator of network activities and for assessment; 4) be purposeful in engaging partners through regular communication and virtual platforms; and 5) invite other disciplines to participate in the use, evaluation, and dissemination of RCN findings, especially in reaching out to diverse participants for RCN activities-explore participation and motivation from the perspective of the diverse audience, not from the perspective of a need for diverse participants. Together, we can work toward our goals of an inclusive, engaging life sciences curriculum.

\section{ACKNOWLEDGMENTS}

We thank all participants of the summit for their contributions to the rich discussions that are the basis for this summary. We thank all organizers and participants of RCN-UBE projects for their energy and valuable contributions that are shared here. This work is supported in part by NSF award DBI-1543972 to G.E.U.

\section{REFERENCES}

Bowne DR, Downing AL, Hoopes MF, LoGiudice K, Thomas CL, Anderson LJ, Gartner T, Hornbach DJ, Kuers K, Machado JL, et al. (2011). Transforming ecological science at primarily undergraduate institutions through collaborative networks. BioScience 61, 386-392.

Donovan S, Eaton CD, Gower ST, Jenkins KP, LaMar MD, Poli D, Sheehy R, Wojdak JM (2015). QUBES: a community focused on supporting teaching and learning in quantitative biology. Lett Biomath 2, 46-55.

Ecological Research and Education Network (2010). EREN home page. http://erenweb.org (accessed 9 March 2016).

Kuh GD (2008). High-Impact Educational Practices: What They Are, Who Has Access to Them, and Why They Matter, Washington, DC: Association of American Colleges and Universities.

National Research Council (2014). Networking field stations for discovery and innovation. In: Enhancing the Value and Sustainability of Field Stations and Marine Laboratories in the 21st Century, Washington, DC: National Academies Press, 33-44.

National Science Foundation (2008). Dear Colleague Letter-Research Coordination Networks-Undergraduate Biology Education. www.nsf.gov/ pubs/2008/nsf08035/nsf08035.jsp (accessed 22 March 2016).

Network for Integrating Bioinformatics into Life Science Education (2016). NIBLSE home page. https://qubeshub.org/groups/niblse (accessed 29 March 2016).

Pentland A (2014). Social Physics: How Good Ideas Spread-The Lessons from a New Science, New York: Penguin.

Quantitative Undergraduate Biology Education and Synthesis (2015). QUBES home page. https://qubeshub.org (accessed 29 March 2016).

Research Coordination Network-Undergraduate Biology Education Summit (2016). RCN-UBE Summit home page. https://qubeshub.org/groups/ rcn_ube2016 (accessed 9 August 2016).

Schmidt B (2015). Foreword. In: Networking for Nerds: Find, Access and Land Hidden Game-Changing Career Opportunities Everywhere, ed. AG Levine, Hoboken, NJ: Wiley, xi-xii.

Simmons JA, Anderson LJ, Bowne DR, Dosch JJ, Gartner TB, Hoopes MF, Kuers K, Lindquist ES, McCay TS, Pohlad BR, et al. (2016). Collaborative research networks provide unique opportunities for faculty and student researchers. Council Undergrad Res Q 36(4), 12-18.

Society for the Advancement of Biology Education Research (2016). SABER home page. https://saber-biologyeducationresearch.wikispaces.com (accessed 29 March 2016).

Zimmerman BJ (2000). Self-efficacy: an essential motive to learn. Contemp Educ Psychol 25, 82-91. 